\title{
Crack propagation in micro-chevron-test samples of direct bonded silicon-silicon wafers
}

\author{
Klaus Vogel, Dirk Wuensch, Alexey Shaporin, Jan Mehner \\ Chemnitz University of Technology, 09107 Chemnitz \\ klaus.vogel@s2005.tu-chemnitz.de,dirk.wnensch@_zfm.tu-chemnitr.de \\ Detlef Billep, Maik Wiemer \\ Fraunhofer Research Institution for Electronic Nano Systems ENAS, Technologie-Campus 3, 09126 Chemnitz;
}

\begin{abstract}
Wafer bonding describes all technologies for joining two or more substrates directly or using certain intermediate layers. Current investigations are focused on so-called low temperature bonding as a special direct bonding technology. It is carried out without intermediate layers and at temperatures below $400{ }^{\circ} \mathrm{C}$. In addition to the wafer materials, the toughness of the bonded interface also depends on the bonding process itself. It can vary for different pre-treatments. Furthermore, an increase of the annealing temperature leads to a higher toughness of the bonded interface.

The fracture toughness is a suitable value to describe the damage behaviour of the bonded interface. Based on a micro-chevron-specimen, the fracture toughness can be determined either numerically or by combining numerical analysis with experimental measurement of the maximum force.

The maximum force is measured during a micro-chevron-test using a Mode I loading. The minimum of the stress intensity coefficient can be determined by a FE-simulation only. One possibility to estimate the stress intensity coefficient is the compliance method. The compliance of the whole specimen increases with a growing crack. The stress intensity coefficient can be directly derived from the simulated compliance and the crack length itself.

The paper is focused on the micro-chevron-test for direct bonded silicon-silicon wafers. Additional to the estimation of dimensionless stress intensity coefficient as a function of geometry, the influence of different pretreatments and annealing temperatures on the measured maximum force are analysed and discussed.
\end{abstract}

KEYwords. Compliance method; FE-analysis; Fracture toughness; Micro-chevron-test; Silicon direct bonding.

\section{INTRODUCTION}

icro Electro Mechanical Systems (MEMS) are applied in a wide industrial range. Their structures become more complex by using different materials in one system. MEMS have at least one typical component size in submillimetre range (smaller than $100 \mu \mathrm{m}$ ) which determines its function [1]. They often consist of two or more 
components and have to be joined by wafer bonding. To ensure their functionality and reliability as well as the quality during the manufacturing process and to provide data for further FE-simulations, significant material parameters are required to characterise such structures.

The behaviour of the structure depends on the bonded interface and the bonding process itself. Wafer bonding describes all technologies for joining two or more substrates directly or using certain intermediate layers. Tab. 1 provides an overview of different bonding technologies $[2,3]$.

\begin{tabular}{|c|c|c|c|}
\hline \multirow{4}{*}{$\begin{array}{l}\text { Without } \\
\text { intermediate layer }\end{array}$} & Bonding & Material & Temperature \\
\hline & $\begin{array}{l}\text { Silicon Direct } \\
\text { Bonding (SDB) } \\
\text { with and without } \\
\text { plasma activation }\end{array}$ & $\begin{array}{l}\text { polished wafers of silicon (wafers } \\
\text { of any orientation and basic } \\
\text { doping with natural, thin } \\
\text { chemical, or thermal oxide ) }\end{array}$ & $\begin{array}{l}\text { low temperature bonding } \\
110 \ldots 400^{\circ} \mathrm{C} \text {, } \\
\text { high temperature bonding } \\
800 \ldots 1100{ }^{\circ} \mathrm{C}\end{array}$ \\
\hline & $\begin{array}{l}\text { Anodic Bonding } \\
\text { (AB) }\end{array}$ & silicon, borosilicate glass & $210 \ldots 450^{\circ} \mathrm{C}$ \\
\hline & $\begin{array}{l}\text { Thermo- } \\
\text { Compression } \\
\text { Bonding }\end{array}$ & materials such as $\mathrm{Cu}, \mathrm{Au}, \mathrm{Ti}$ & $300 \ldots 400^{\circ} \mathrm{C}$ \\
\hline \multirow[t]{3}{*}{$\begin{array}{l}\text { With } \\
\text { intermediate layer }\end{array}$} & Eutectic Bonding & substrate and $\mathrm{Au}$ & $\begin{array}{l}\text { Au-Si } 363^{\circ} \mathrm{C} \\
\text { Au-Sn } 280^{\circ} \mathrm{C} \\
\mathrm{Cu}-S n 350^{\circ} \mathrm{C} \\
\text { Al-Si } 577^{\circ} \mathrm{C}\end{array}$ \\
\hline & Adhesive Bonding & $\begin{array}{l}\text { silicon / glass, plastics / } \\
\text { polymers, special materials } \\
\text { (ceramics, metals, PCB, tapes), } \\
\text { epoxy resins, UV adhesives, } \\
\text { ceramic adhesives, photo resists }\end{array}$ & room temperature $\ldots 300^{\circ} \mathrm{C}$ \\
\hline & Glas Frit Bonding & substrate + glass frit - substrate & $430^{\circ} \mathrm{C}$ \\
\hline
\end{tabular}

Table 1: Overview of bonding technologies.

In particular, direct bonding has a major advantage within the packaging process because no additional intermediate layers like in eutectic or adhesive bonding are required. This technology uses the formation of covalent bonds for joining two clean and smooth surfaces before annealing them to temperatures up to $1100{ }^{\circ} \mathrm{C}$. For many applications these high temperatures have to be reduced. This reduction can be done using surface activating procedures prior to bonding such as low pressure plasma or ion beam treatment. The surface can be activated by a variety of low pressure plasma modes. Besides remote and sequential plasma, a commonly used mode is the reactive ion-etching (RIE). The RIE-system operates in a fine vacuum with typical ranges of $10 \mathrm{~Pa}$ to $20 \mathrm{~Pa}$ with a continuous gas flow in the reactor chamber. An operation frequency of $13.56 \mathrm{MHz}$ is used. Compared to conventionally bias voltage the ions are only accelerated by approximately $100 \mathrm{~V}[4]$.

To understand the mechanism of a plasma treatment on the surface, it is necessary to explain the procedure of the hydrophilic fusion bonding. The process includes the pre-bonding at room temperature and an annealing at high temperatures in order to develop strong covalent bonds. For hydrophilic direct bonding, the bonding process at room temperature is caused by hydrogen bonds between the water molecules located on the opposing wafer surfaces after contacting the wafers. The bonding strength especially depends on the number of hydrogen bonds and therefore on the number of silanol groups ( $\mathrm{Si}-\mathrm{OH})$ and water molecules. A subsequent heat treatment (annealing) is carried out, to increase the bond strength between the two wafers. Thereby, the water molecules diffuse out either along the interface to the outside or through the native oxide to the bulk material. The wafers move closer towards each other and form hydrogen bonds between the silanol groups. By increasing the temperature the opposing silanol groups react with each other to form covalent siloxane bonds [5-8]. 
The fracture toughness is one of the suitable values to describe the damage behaviour of the bonded interface. Based on a micro-chevron-specimen, the fracture toughness of this specimen can be determined numerically and experimentally. The experimental determination can be executed by combining experiment with numerical analysis.

\section{THEORY}

he analysed samples consist of two single chips bonded together. Because they have a quadratic footprint, their width $w$ and thickness $t$ are equal $[9,10]$. The analysis is focused on specimens with both a width and a thickness of $10 \mathrm{~mm}$, Fig. 1.

The height of the specimen depends on the height of the unstructured wafer $h_{w 1}$ and the height of the structured wafer $h_{w 2}$ as well as the structure height, Fig. 2. While the height of the structured chip is kept constant, the height of the unstructured chip varies for different material combinations.

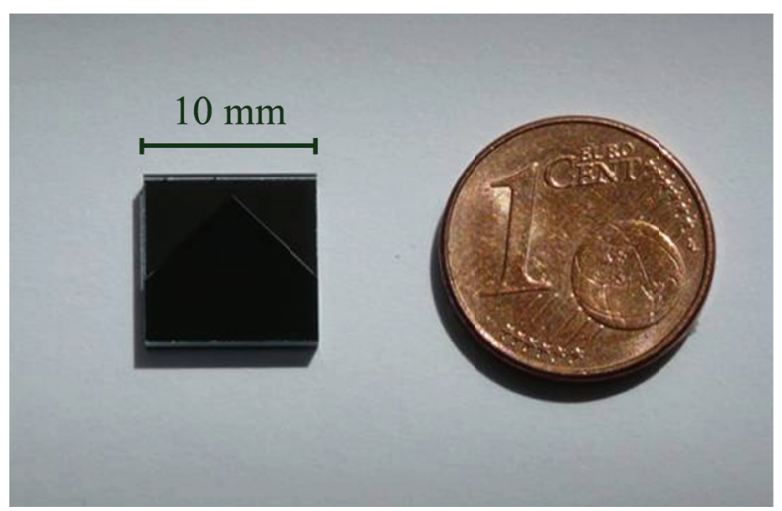

Figure 1: Geometry of a micro-chevron-specimen compared to an one cent coin.

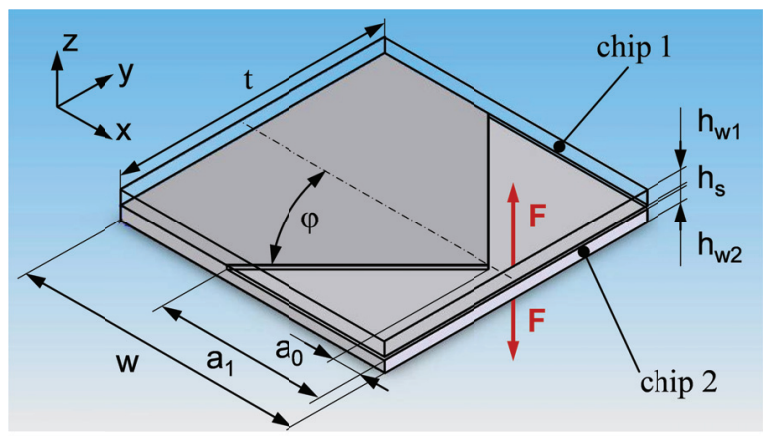

Figure 2: Micro-chevron-specimen prepared from a processed wafer.

The bonded chip is loaded perpendicular to the $x-y$-plane in front of the sharp notch. The lifting of the crack fronts leads approximately to a Mode I crack opening. So the fracture toughness $K_{I C}$ can be calculated against the geometrical parameters width and thickness by

$$
K_{I C}=\frac{F_{M A X}}{t \cdot \sqrt{w}} \cdot Y_{M I N}
$$

While the maximum force $F_{M A X}$ can be measured during a tensile test, the minimum of the stress intensity coefficient $Y_{M I N}$ is determined by FE-simulation.

One possibility to estimate the stress intensity coefficient is the compliance method. It combines experiment with numeric analysis. With an extension of the crack length, the compliance of the specimen increases too. By keeping the 
displacement $u_{z}$ constant, the reaction forces $F$ are simulated subjected to a well defined crack propagation. For different relative crack lengths

$$
\alpha=\frac{a}{w} \quad \alpha_{0}=\frac{a_{0}}{w} \quad \alpha_{1}=\frac{a_{1}}{w}
$$

the compliance $C(\alpha)$ can be interpolated, using the equation

$$
C(\alpha)=\frac{u_{z}}{F(\alpha)}
$$

After scaling the compliance with the thickness $t$ of the sample, the Young's modulus $E$ and the Poissons ratio $v$ of silicon [10]

$$
C^{\prime}(\alpha)=\frac{E \cdot t}{1-v^{2}} \cdot C(\alpha)
$$

the function of the stress intensity coefficient can be determined

$$
Y(\alpha)=\sqrt{\frac{1}{2} \cdot \frac{d}{d \alpha} \cdot C^{\prime}(\alpha) \cdot \frac{\alpha_{1}-\alpha_{0}}{\alpha-\alpha_{0}}}
$$

Its minimum, the stress intensity coefficient $Y_{M I N}$ can be calculated. Inserting $Y_{M I N}$ in Eq. (1) leads to the fracture toughness.

\section{EXPERIMENT}

I

$\mathrm{n}$ addition to the numerical determination of the stress intensity coefficient the maximum force is measured during a micro-chevron-test. Before carrying out the experiments, the samples have to be prepared. Their preparation can be divided into the following steps. First, all Si wafers are RCA-cleaned before applying the low pressure plasma. Then the particle concentration has to be reduced within a spin dryer, after rinsing the wafers in deionised water. The wafers are pre-bonded at room temperature afterwards. Before dicing the wafer stacks into specimens, they have to be annealed for 6 hours. The annealing conditions can vary between different batches. To initiate the force, two studs are glued on the top and the bottom of the specimen, Fig. 3. Before starting the experiment, the specimen has to be preloaded.
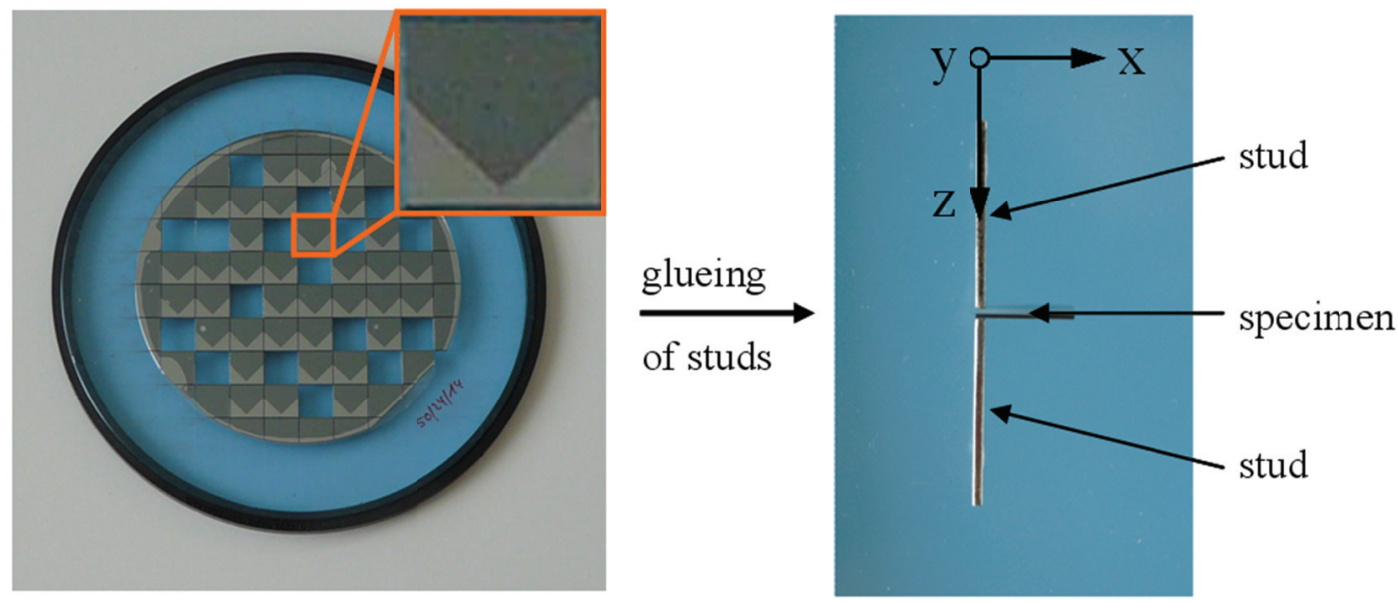

Figure 3: Preparation of the micro-chevron-samples. 
The experiment is carried out displacement controlled. Therefore a micro-chevron-tester is used to measure the reaction forces subjected to the applied deflection. When the crack length a reaches its critical value, the measured force converges toward the maximum force $F_{M A X}$ before decreasing again. When the crack length exceeds the value $a_{1}$ the stable crack propagation becomes instable and the specimen fails. The measured force becomes almost zero again.

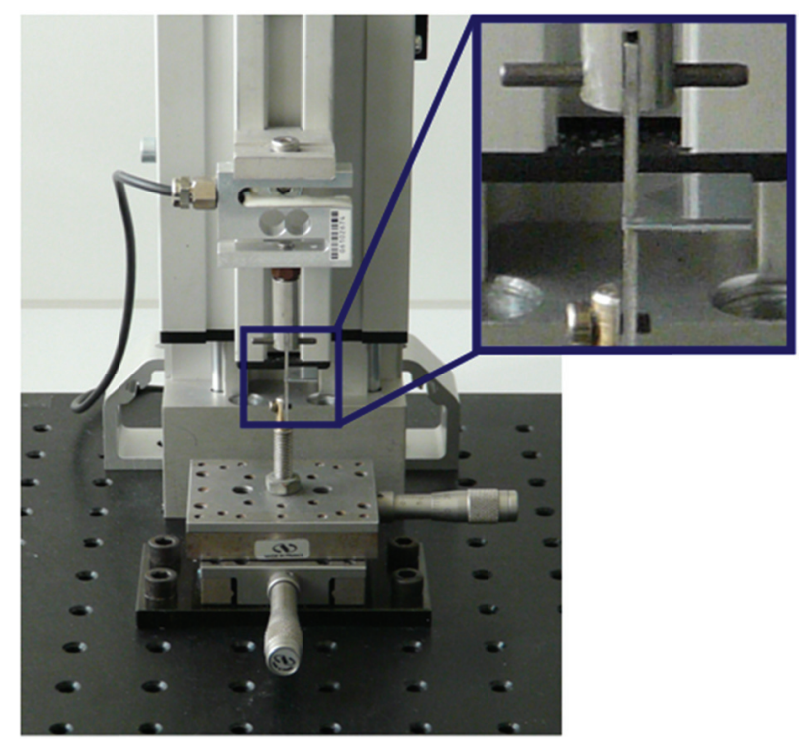

Figure 4: Experimental setup for the measurement of the maximum force.

The first series of experiments focus on the heat treatment of the specimens. All specimens have no further pretreatment. In that case, no plasma activation has been carried out. Their annealing temperatures vary between $40{ }^{\circ} \mathrm{C}$ and $150^{\circ} \mathrm{C}$, Fig. 5.

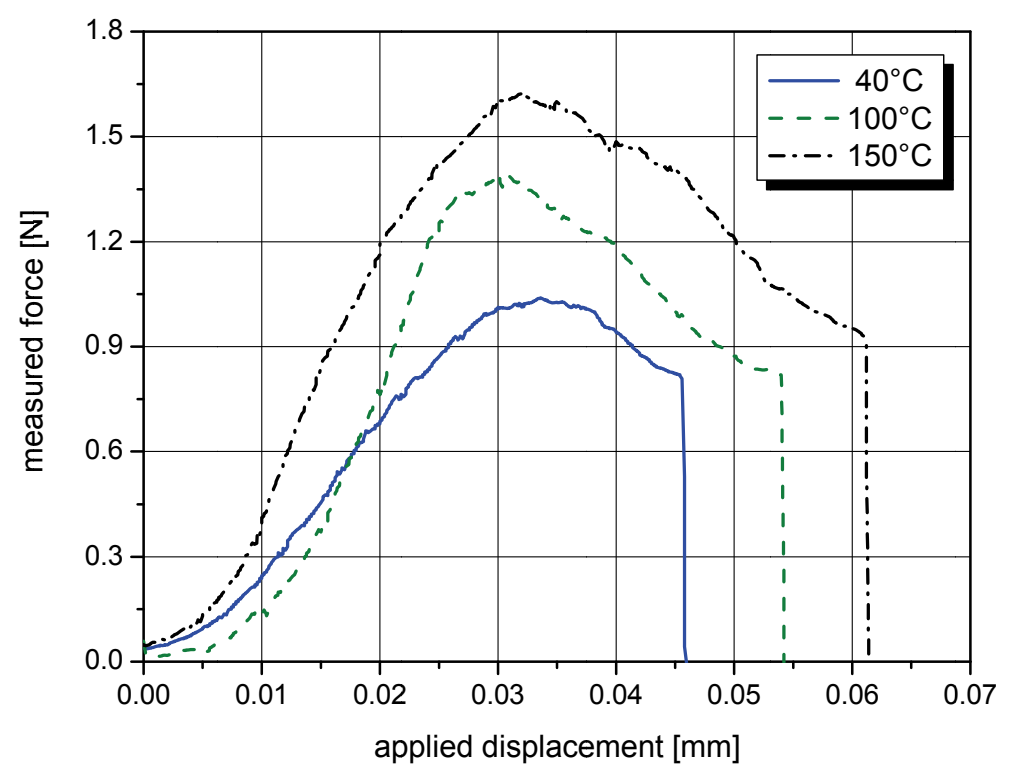

Figure 5: Force displacement curves for different heat treatments (annealing temperatures).

An increase of the annealing temperature leads to an increase of the measured maximum force. For specimens with no further pre-treatment and an annealing temperature of $150{ }^{\circ} \mathrm{C}$, the measured maximum force is about $35 \%$ higher than the one for annealing temperatures of only $40{ }^{\circ} \mathrm{C}$. 
In addition to the heat treatment, the influence of the pre-treatment has to be characterised as well. As already mentioned in the introduction, the surface activation can be carried out using different plasmas and different process gases. The duration of the plasma treatment can also vary. Three different pre-treatments are compared in the present paper. The reference specimens have no plasma activation. Moreover, plasma activations in oxygen ( $\mathrm{O}_{2}$-plasma) and in nitrogen plasma ( $\mathrm{N}_{2}$-plasma) have been taken into account. The duration for the plasma treatment for the activated stacks was both five minutes. The annealing temperature of $100{ }^{\circ} \mathrm{C}$ was the same for all three stacks, too. The maximum forces of these samples vary between $1.38 \mathrm{~N}$ and $4.26 \mathrm{~N}$, Fig. 6.

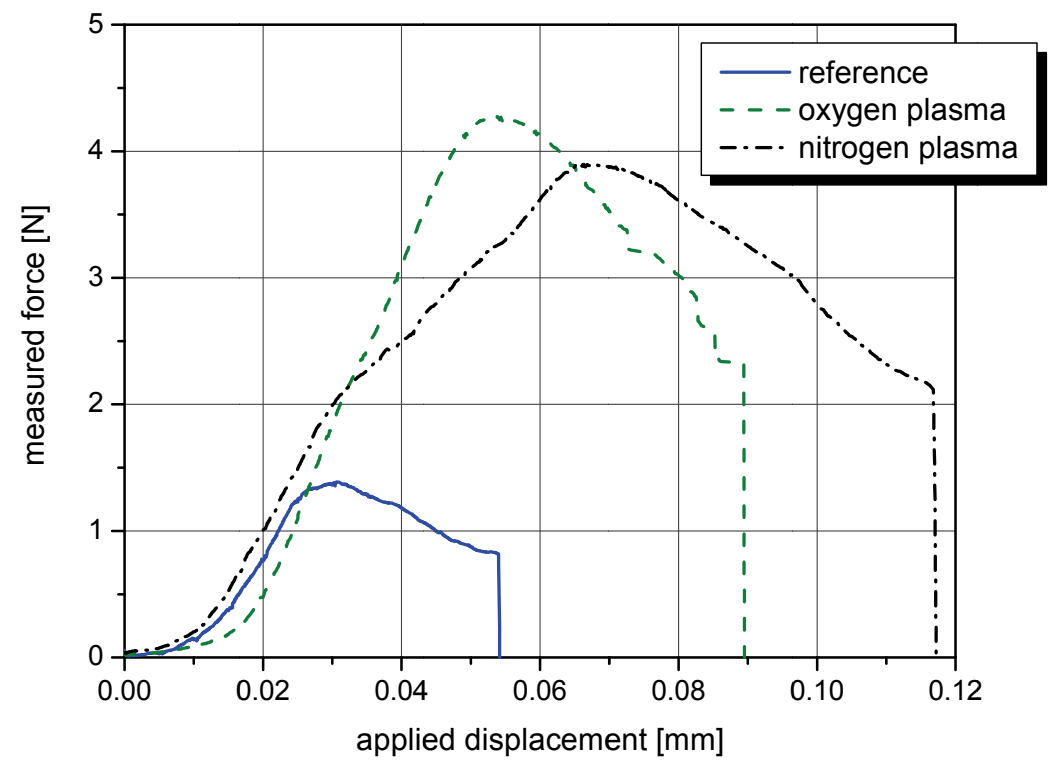

Figure 6: Force displacement curves for different pre-treatments.

For specimens with plasma activation, the measured maximum forces are approximately three times higher than the once without any plasma activation. The maximum forces for samples activated in nitrogen plasma are a little bit smaller than for oxygen plasma.

\section{RESULTS}

7 he variation of geometries and bond parameters leads to different functions for the stress intensity coefficient and therefore to different fracture toughness.

By keeping the structure height $b_{S}$ constant, and changing the position of the structure by using two structured wafers (Si-Si geometry 2) instead of a sample consisting of an unstructured and a structured chip (Si-Si geometry 1), only a negligible variation of $Y(\alpha)$ can be observed, Fig. 7.

The variation of the wafer height leads to a significant deviation between the functions and minima of the stress intensity coefficients. So the values of $Y(\alpha)$ decrease with increasing wafer height.

Based on the geometry, the minima of the stress intensity coefficient and the maximum force recorded during experiment, the fracture toughness can be determined. Using the same geometries the fracture toughness only depends on the parameters of the manufacturing process. Smaller fracture toughnesses can be observed for lower annealing temperatures. A heat treatment at higher temperatures leads to higher maximum forces and also to higher fracture toughnesses. The measured maximum force and the fracture toughness of the specimens can be significantly increased by activating the surfaces of the wafers, either in oxygen or nitrogen plasma, before bonding, Fig. 8.

Applying the same annealing temperature, the maximum force and therefore the fracture toughness of oxygen activated specimens is higher than the one of samples with any other pre-treatment. The deviation between oxygen and nitrogen activated specimens varies for different annealing temperatures. Furthermore, the fracture toughness of plasma activated samples with an annealing temperature of $40{ }^{\circ} \mathrm{C}$ is much higher than the one of non-activated specimens at higher temperatures. 


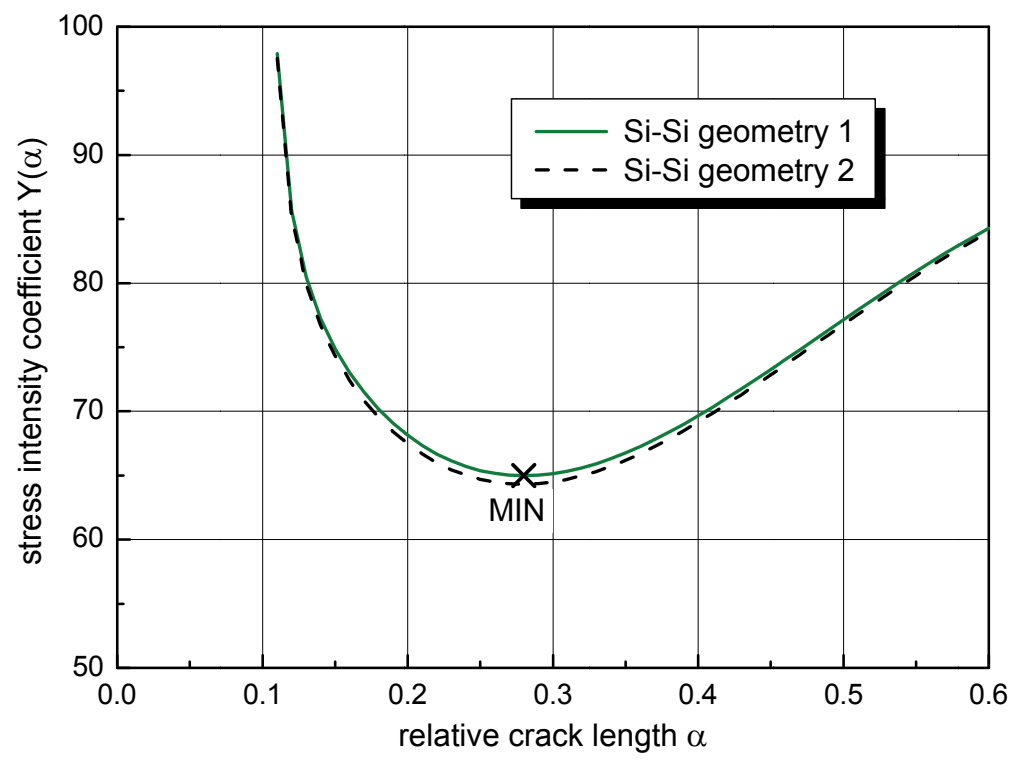

Figure 7: Estimation of dimensionless stress intensity coefficient as a function of geometry.

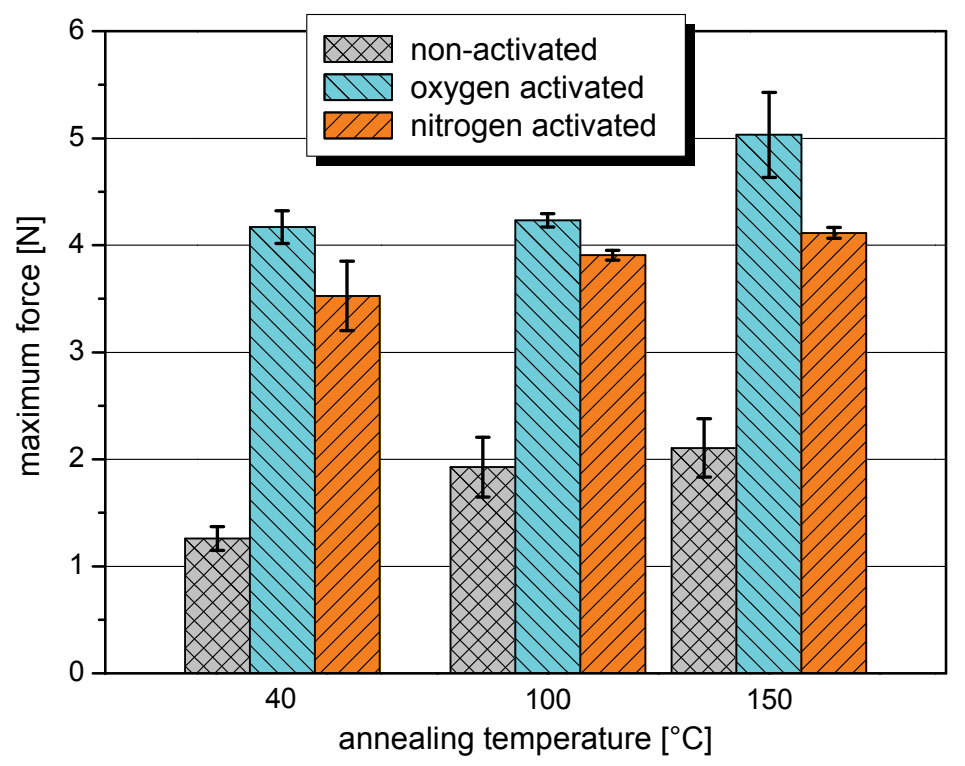

Figure 8: Maximum forces for different pre and heat treatments.

\section{CONCLUSIONS}

$\mathrm{T}$

he compliance method is a suitable approach to estimate the fracture toughness of direct bonded wafers. While the influence of the specimen geometry is considered during the calculation of stress intensity coefficient, the pretreatment and the bonding temperature itself directly affect the measured maximum force. The use of plasma activated wafers during the manufacturing process leads to significantly reduced annealing temperatures without any loss of bonding strength. 
Currently, the stress intensity coefficient is calculated using another numeric approach, the energy release rate, to verify the results of the compliance method. In addition to the numeric calculations the measurement of the crack length depending on the applied load will be carried out in future.

\section{REFERENCES}

[1] G. Gerlach, W. Doetzel: Introduction to microsystem technology. John Wiley \& Sons, Ltd., (2008).

[2] M. Wiemer, J. Froemel, T. Gessner, T. Otto, In: The World of Electronic Packaging and System Integration, Ed. by B. Michel, R. Aschenbrenner, (2004) 307.

[3] M. Wiemer, J. Froemel, M. Haubold, C. Jia, D. Wuensch, T. Gessner, ECS Transactions, 16(8) (2008) 81.

[4] D. Wuensch, B. Mueller, M. Wiemer, T. Gessner, H. Mischke, In: MNI Proceedings, Darmstadt, (2010) 66.

[5] Q. Tong, U. Goesele, Semiconductor wafer bonding, John Wiley \& Sons, Ltd., New York, (1999).

[6] M. Wiemer, T. Otto, T. Gessner, K. Hiller, K. Kapser, H. Seidel, J. Bagdahn, M. Petzold, Materials Research Society, 682E (2001).

[7] A. Ploessl, G. Kraeuter, Materials Science and Engineering, R25 (1999) 1.

[8] T. Suni, Direct wafer bonding for MEMS and microelectronics, PhD thesis, Helsinki University of Technology, (2006).

[9] J. Bagdahn, A. Ploessl, M. Wiemer, M. Petzold, Electrochemical Society, (2001) 218.

[10] D. Munz, R.T. Bubsey, J.E. Srawley, Int. J. of Fracture, 16(4) (1980) 359. 